\title{
Vitamin D and human health: more than just bone
}

John H. White

In their Perspective article (Common misconceptions about vitamin D-implications for clinicians. Nat Rev. Endocrinol. doi:10.1038/nrendo.2013.75), ${ }^{1}$ Rosen and Taylor have provided a primer on the state of vitamin D research. However, the analyses presented of the current literature are not balanced. The conclusions of the authors largely reflect those of the 2011 Institute of Medicine (IOM) report, ${ }^{2}$ which immediately created a controversy, leading to published rebuttals. ${ }^{3}$ The IOM report was controversial in part because of its very conservative recommendations for dietary intakes, its conclusions of insufficient evidence for any role of vitamin D in nonbone health, and the way it presented evidence for the potential harm associated with circulating 25-hydroxyvitamin D levels.

Notably, considerable debate surrounds potential roles of vitamin $\mathrm{D}$ in nonbone indications including cancer prevention and control of immune system function. The authors conclude that "effects of vitamin $\mathrm{D}$ on nonbone disorders is currently best described as consisting of hypotheses of emerging interest". ${ }^{1}$ They also claim that vitamin D supplementation has not been shown to prevent infections. In fact, several randomized placebo-controlled trials have been published providing evidence for vitamin D supplementation of deficient populations in preventing a variety of infections. In addition to the (highly cited) results of a trial published in 2010 concluding that vitamin D supplementation reduced the risk of seasonal influenza infections in a paediatric population, ${ }^{4}$ recent studies have provided evidence for a beneficial role of vitamin $\mathrm{D}$ supplementation of populations at risk for upper respiratory tract or ear infections, either because of severe vitamin D deficiency or a history of recurrent infections. ${ }^{5,6}$ By contrast, one study that produced a negative result involved a healthy population with baseline 25-hydroxyvitamin D levels of $73 \mathrm{nmol} / \mathrm{l}^{7}$ The authors also discuss the emerging evidence for a U-shaped curve of risk associated with 25-hydroxyvitamin D levels and focus largely on the potential risks associated with excessive vitamin D intake, an area that should not be ignored. They cite a recent review ${ }^{8}$ suggesting that there may be an increase in prevalence of certain cancers associated with high serum 25-hydroxyvitamin D levels (>75 nmol/l). However, $\mathrm{U}$-shaped curves have two sides, and the IOM recommends that the vitamin $\mathrm{D}$ needs of $\sim 50 \%$ of the population can be met with 25-hydroxyvitamin D levels of 30-40 nmol/l, whereas those of most of the remainder require 40-50 $\mathrm{nmol} / 1$. If followed, these recommendations would place the entire population solidly within the left-hand side of the U-shaped curve of cancer risk. It can therefore be argued that one of the misconceptions arising from conservative estimates of recommended vitamin D intake in the IOM report is that less vitamin D does no harm.
Departments of Physiology and Medicine, McGill University, 3655 Drummond Street, Room 1112, H3G 1Y6 Montreal, QC, Canada. john.white@mcgill.ca

Competing interests

The author declares no competing interests.

1. Rosen, C. J. \& Taylor, C. L. Common misconceptions about vitamin D-implications for clinicians. Nat. Rev. Endocrinol. 9, 434-438 (2013).

2. Institute of Medicine. Dietary Reference Intakes for Calcium and Vitamin D (eds Ross, A. C., Taylor, C. L., Yaktine, A. L. \& Del Valle, H. B.) (National Academies Press, Washington DC, 2011).

3. Holick, M. F. et al. Evaluation, treatment, and prevention of vitamin D deficiency: an Endocrine Society clinical practice guideline. J. Clin. Endocrinol. Metab. 96, 1911-1930 (2011).

4. Camargo, C. A. Jr. et al. Randomized trial of vitamin $D$ supplementation and risk of acute respiratory infection in Mongolia. Pediatrics 130, 561-567 (2012).

5. Bergman, P. et al. Vitamin $\mathrm{D}_{3}$ supplementation in patients with frequent respiratory tract infections: a randomised and double-blind intervention study. BMJ Open 2, e001663 (2012).

6. Marchisio, P. et al. Vitamin D supplementation reduces the risk of acute otitis media in otitisprone children. Pediatr. Infect. Dis. J. http:// dx.doi.org/10.1097/INF.0b013e31829be0b0.

7. Murdoch, D. R. et al. Effect of vitamin D3 supplementation on upper respiratory tract infections in healthy adults: the VIDARIS randomized controlled trial. JAMA $\mathbf{3 0 8}$, 1333-1339 (2012).

8. Mayne, S. T., Ferrucci, L. M. \& Cartmel, B. Lessons learned from randomized clinical trials of micronutrient supplementation for cancer prevention. Annu. Rev. Nutr. 32, 369-390 (2012). 